\title{
Lattice Boltzmann Simulation of Thermal Microflows with Heterogenuous Catalysis
}

\author{
Sauro Succi \\ Istituto Applicazioni Calcolo, viale del Policlinico 137, 001 61, Roma, Italy, \\ succi@iac.rm.cnr.it, \\ http://www.iac.rm.cnr.it
}

\begin{abstract}
The effects of temperature dynamics on the conversion efficiency of a model micro-reactor are computed by means of a Lattice Boltzmann simulation coupled to a finite-difference treatment of chemical species and temperature transport.
\end{abstract}

\section{Introduction}

The dynamics of reactive microflows is a broad interdisciplinary subject with many applications in material science, biology and engineering [1]. Of particular relevance for practical applications is the case in which the chemical activity takes place only at the solid boundaries of the fluid domain (heterogeneus catalysis). Since the rate of chemical consumption at the solid walls determines the overall effciency of the micro-reactor, an accurate description of the fluid interaction with the reactive wall is of great importance.

The fluid-wall interaction depends on a number of important factors, such as the geometrical conformation of the wall, and, at a smaller scale, the interatomic interactions between solid and fluid molecules. On the other hand, since catalytic reactions are normally governed by activated processes, they generally exhibit a non-analytic, Arrhenius-like dependence on the wall temperature. As a result, an accurate description of temperature dynamics is central to the design of micro-reactors. One of the major difficulties in modeling these phenomena traces to the multiple space-time scales associated with the interaction between the physics of fluids and chemical reactions. In macroscopic devices, the typical example of such multiscale coupling is turbulent combustion: owing to the energy cascade from large to small scales, highly turbulent flows develop active excitations at a time-scale comparable with that of chemical reactions. In microdevices, such as those addressed in this paper, turbulence is generally not active, but hydrodynamic and chemical time scales can nonetheless come close together due to reduced size of the device. Again, thermal effects play a major role since even minor temperature fluctuations in the vicinity of the activation temperature can generate wide excursions of the reactive timescales.

A particularly desirable feature of numerical models aimed at describing these multiscale phenomena is space-time accuracy. Several powerful techniques have 
been developed over the years [2]. Among others, a particularly simple and effective strategy is based on the lattice Boltzmann method [3]. One of the merits of this method is to tick at a very small time-scale as compared to fluid solvers, (typically ten time faster) thereby permitting a more direct coupling between the bulk and wall physics. Such capabilities of the LB method have been explored and demonstrated in the recent past for isothermal microflows [4-6].

In this paper, we extend this type of approach to the case of reactive microflows with temperature dynamics.

\section{The Mathematical Model}

In the present model, the bulk flow is described by standard Navier-Stokes equations for quasi-incompressible flows, coupled to a transport equation for the pollutant concentration and for the temperature field. In equations,

$$
\begin{array}{r}
\partial_{t} \boldsymbol{u}+\boldsymbol{u} \cdot \nabla \boldsymbol{u}=-\nabla P+\nu \Delta \boldsymbol{u} \\
\partial_{t} C_{s}+\boldsymbol{u} \cdot \nabla C_{s}=D_{s} \Delta C_{s} \\
\partial_{t} T+\boldsymbol{u} \cdot \nabla T=\kappa \Delta T
\end{array}
$$

where $\boldsymbol{u}$ is the flow speed, $P$ the fluid pressure and $\nu$ the kinematic viscosityi, and fluid density is set to unity. In the second equation, $C_{s}$ is the concentration of the $s$-th species, and $D_{s}$ is the corresponding mass diffusivity. Finally, $T$ represents the fluid temperature and $\kappa$ the thermal diffusivity. Since we deal with microflows where bulk time-scales are comparable with the time-scale of wall reactions, a dynamic treatment of boundary conditions is required, as we shall discuss in the sequel.

\subsection{Reactive Thermal Boundaries}

The physical scenario we wish to describe is a thermal flow conveying a pollutant species. Upon hitting the walls of the domain (a box of height $H$, width $W=H$ and length $L$ ), the pollutant molecules undergo a first order catalytic reaction of the form:

$$
\begin{array}{r}
\frac{d C_{f}}{d t}=-\left(C_{f}-C_{s}\right) / \tau_{w}+J \\
\frac{d C_{s}}{d t}=\left(C_{f}-C_{s}\right) / \tau_{w}-C_{s} / \tau_{c}
\end{array}
$$

where $C_{s}, C_{f}$ are the pollutant concentrations at a solid cell and its fluid neighbor cells respectively. The coefficients $\tau_{w}, \tau_{c}$ are empirical time-scales for fluid-to-wall mass transfer and catalytic reaction respectively. Temperature at the solid wall is assumed to obey a similar set of equations:

$$
\begin{array}{r}
\frac{d T_{f}}{d t}=\left(T_{f}-T_{s}\right) / \theta_{w}+Q \\
\frac{d T_{s}}{d t}=\left(T_{f}-T_{s}\right) / \theta_{w}-T_{s} / \theta_{c}
\end{array}
$$


Here, $T_{f}$ and $T_{s}$ are the fluid and wall temperatures. The coefficients $\theta_{w}$ and $\theta_{c}$ are typical time-scales for thermal transport. Finally, $J=-D \nabla C$ and $Q=$ $-\kappa \nabla T$ are the mass and heat fluxes from the bulk flow.

These equations are applied to all buffer cells placed at $z=0$ and $z=H+1$ and serve as a dynamic boundary condition for the pollutant concentration as well as for the flow temperature.

Temperature dynamics affects the pollutant concentration via an Arrhenius dependence of the reaction rate on the solid wall temperature:

$$
\tau_{c}=\tau_{0} e^{+T_{a} / T_{s}}
$$

where $T_{a}$ is the activation temperature of the chemical reactioni and $\tau_{0}$ fixes the fastest chemical timescale (corresponding to $T_{s} \rightarrow \infty$ ).

The above dynamical system is driven and sustained by the bulk-to-wall mass and thermal fluxes $J, Q$. The time evolution of these fluxes is controlled by the bulk transport coefficients, and we are primarily interested into the asymptotic values of the wall quantities which are attained once the fluxes settle down to their asymptotic value. The steady-state solution of the above system of ordinary differential equations reads as follows:

$$
\begin{array}{r}
C_{f}^{\infty}=J^{\infty}\left(\tau_{w}+\tau_{c}\right) \\
C_{s}^{\infty}=J^{\infty} \tau_{c} \\
T_{f}^{\infty}=Q^{\infty}\left(\theta_{w}+\theta_{c}\right) \\
T_{s}^{\infty}=Q^{\infty} \theta_{c}
\end{array}
$$

From these equations, we see that the highest efficiency is attained when $\tau_{c}=0$ (infinitely fast chemistry limit).

\section{The Numerical Model}

The Navier-Stokes equations are solved by the Lattice Boltzmann equation, whereas the evolution of the pollutant concentration and temperature field are handled with a finite-difference Lax-Wendroff scheme [7].

The lattice Boltzmann equation is solved in its LBGK form [8]:

$$
f_{i}\left(\mathbf{x}+\delta_{t} \mathbf{c}_{i}, t+\delta_{t}\right)-f_{i}(\mathbf{x}, t)=-\omega \delta_{t}\left[f_{i}(\mathbf{x}, t)-f_{i}^{e}(\mathbf{x}, t)\right]
$$

where $f_{i}(\mathbf{x}, t) \equiv f\left(\mathbf{x}, \mathbf{v}=\mathbf{c}_{i}, t\right), i=1, n$, is the probability of finding a particle at lattice site $\mathbf{x}$ at time $t$, moving along the lattice direction defined by the discrete speed $\mathbf{c}_{i}$ and $\delta_{t}$ is the time unit. For the present study, a three-dimensional, nineteen-speed model is used.

The left-hand side of this equation represents the molecular free-streaming, whereas the right-hand side represents molecular collisions via a simple relaxation towsubards local equilibrium $f_{i}^{e}$ (a local Maxwellian expanded to second order in the fluid speed) in a time lapse of the order of $\omega^{-1}$. This relaxation time fixes the fluid kinematic viscosity as $\nu=c_{s}^{2}(1 / \omega-1 / 2)$, (in lattice units $\delta_{t}=1$, 
$\delta_{x}=1$, where $c_{s}$ is the sound-speed of the lattice fluid, $1 / \sqrt{3}$ in the present work. In order to recover fluid dynamics, the set of discrete speeds must be chosen such that mass, momentum and energy conservation are fulfilled. Once this is secured, the fluid density $\rho=\sum_{i} f_{i}$, and speed $\mathbf{u}=\sum_{i} f_{i} \mathbf{c}_{i} / \rho$ evolve according to the Navier-Stokes equations of fluid-dynamics.

The advantages of the kinetic representation are computational efficiency, easy accomodation of irregular boundary conditions, and flexible inclusion of additional physics at mesoscopic scale. In this work, we shall appeal basically to the former of these properties. In particular, we refer to the fact that the Lattice Boltzmann method provides the pressure field 'on the fly', with no need of solving the Poisson problem, usually the computationally most intensive task in explicit methods for fluid flows. The LB method can be extended in such a way as to describe thermal transport phenomena [9]. However, since this generalization does not yet offer the same degree of robustness of its athermal counterpart, we resolve for evolving the temperature field with a separate finite-difference LaxWendroff scheme [7]. Since this model and the corresponding algorithms have been thoroughly discussed in previous papers, for further details we refer the reader to the original publications $[7,10]$.

\section{Theoretical Efficiency}

The major macroscopic parameter of the micro-reactor is the conversion efficiency, defined as the fraction of injected pollutant which is absorbed at the catalytic walls. Mathematically, this is defined as:

$$
\eta=1-\frac{\Phi_{\text {out }}}{\Phi_{\text {in }}}
$$

where $\Phi_{\text {in }}, \Phi_{\text {out }}$ are the fluxes of pollutant at the inlet and outlet sections of the micro-reactor respectively, $\Phi(x)=\sum_{y z} C(x, y, z) u(x, y, z)$. The conversion efficiency of a smooth micro-reactive channel can be estimated as follows [4]:

$$
\eta=1-e^{-\frac{L}{2 H} \frac{\lambda(D a)}{P e}}
$$

where $P e \equiv \frac{U H}{2 D}$, is the Péclet number, measuring advection versus diffusion time scales, and $D a \equiv \frac{H^{2}}{4 D \tau}$ is the diffusive Damkoheler number measuring diffusion versus chemical time-scales (here $\tau=\tau_{w}+\tau_{c}$ ). In the above $\lambda=H / 2 l_{z}$, where $l_{z}$ is the transversal wavelength of the concentration profile. By imposing zerospeed at the boundary, we obtain the following algebraic constraint:

$$
\lambda^{2}=\frac{2 \delta}{H} D a \cos (\lambda)
$$

where the lattice spacing $\delta$ has been reintroduced for the sake of dimensional clarity. The above relation shows that $\lambda$ is an increasing function of the Damkoheler number, which meets the intuitive notion that high Damkoheler numbers (fast chemistry) associate with high efficiency. Similarly, high Péclet numbers 
(fast flows) spell poor efficiency, simply because "fast" flows give the pollutant "no time" to react. The role of temperature dynamics in this picture is clear: heat diffusion from the bulk flow raises the wall temperature, hence the catalytic reaction rate. Within the analytic formula given above, this effect should be entirely taken into account by the proper value of the total fluid-wall interaction scale $\tau\left(T_{s}\right)=\tau_{w}+\tau_{c}\left(T_{s}\right)$, withe $\tau_{c}$ given by the expression (8).

\section{$5 \quad$ Numerical Results}

We simulate a fluid initially at rest and with a temperature $T_{f}(0)=T_{w}(0)=$ $T_{0}$. The pollutant is continuously injected at the inlet section with a constant value of the concentration $C_{i n}=1$. From the same inlet section, hot fluid at a temperature $T_{i n}>T_{0}$ is also continuously injected. The boundary conditions are as follows. Fluid flow: periodic boundary conditions at inlet/outlet, and zerospeed conditions on the remaining boundaries. Pollutant: constant injection at $C=1$ at the inlet section, and open boundary at the outlet (molecules which make it to the outlet are lost to the 'environment'). At the remaining boundaries, reactive conditions are imposed. Temperature: same as the pollutant. The main parameters of the simulation are: $L=8 \mathrm{~mm}, H=2 \mathrm{~mm}$, and a flow speed $U=10-50 \mathrm{~m} / \mathrm{s}$. On a $80 \times 20 \times 20$ grid, each lattice spacing corresponds to 0.1 $\mathrm{mm}$ in size, yielding a lattice time step of about $0.3 \mu \mathrm{s}$. The other parameters are (in lattice units): pollutant mass diffusivity, $D=0.01$, fluid kinematic viscosity, $\nu=0.01$, and thermal diffusivity $\kappa=0.01$. This yields the following timescales: $t_{D} \equiv H^{2} / 4 D=10000, t_{A} \equiv L / U=800$. The chemical time-scales are varied in the following range: $\tau_{w}=10-1000, \tau_{c}=10-1000$, whereas the thermal timescales are kept frozen to $\theta_{w}=\theta_{c}=100$. This corresponds to a fastchemistry scenario in which catalytic processes occurr at comparable or shorter scales than the fastest fluid-dynamic process (advection). Note that the LBE timestep $\left(\delta_{t}=1\right)$ is always much shorter than the fastest chemical timescale.

\subsection{Isothermal Walls: $T_{s}=$ const}

As a first series of simulations, we have considered the case of a constant wall temperature $T_{s}=$ const. Here and throughout the hot fluid at the inlet has a temperature $T_{i n}=1$. Three set of simulations with $T_{s} / T_{a}=0.1-10$ for $\tau_{c}=10^{1}, 10^{2}, 10^{3}$ and $\tau_{w}=10^{2}$, have been performed. The results are shown in Figure 1.

From this figure, the expected steep dependence on the wall temperature is well visible. Clearly, the sharpest rise of efficiency occurs in the proximity of $T_{a}$ (basically three orders of magnitude in going from $T_{s}=0.1 T_{a}$ to $T_{s}=T_{a}$ ), any further raise of $T_{s}$ resulting in very marginal gains of efficiency. These data are in qualitative agreement with the analytical expression (13) (solid points), showing that indeed the main effect of wall temperature is absorbed by the Arrhenius dependence of the chemical timescale $\tau_{c}$ on the wall temperature. To further test the analytical expression, we have also varied the mass-transfer 


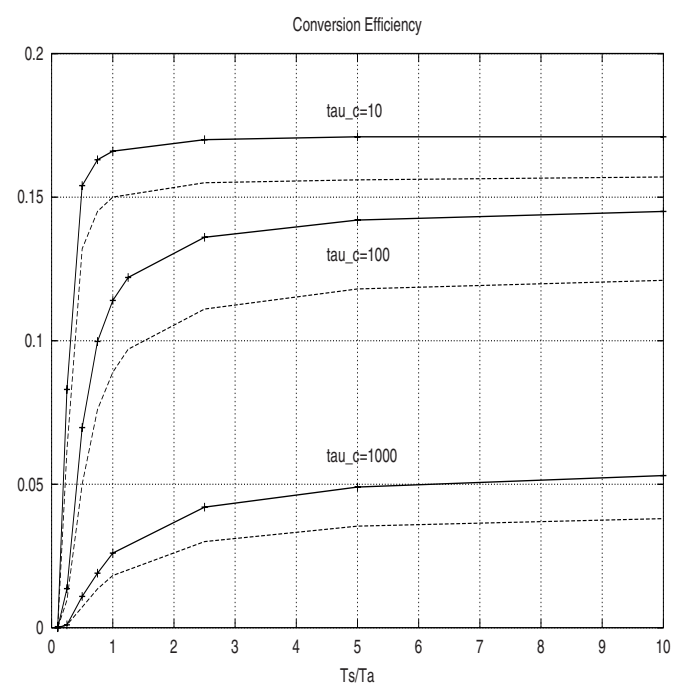

Fig. 1. Conversion efficiency as a function of the solid temperature $T_{s}$ (in units of activation temperature $T_{a}$ ) for three different chemical timescales $\tau_{c}=10,100,1000$ (top to bottom). The absorption time scale is $\tau_{w}=100$, whereas the mass and heat diffusivity are $D=\kappa=0.01$. The thin lines indicate the values obtained by the analytical expression 13 .

timescale $\tau_{w}$, using the values $\tau_{w}=0.1,0.001$. The qualitative effect of raising $\tau_{w}$ is to enhance fluid-wall transfer, hence increase the conversion efficiency. The resulting conversion efficiency is shown in Figure 2.

Indeed, the conversion efficiency is significantly increased, but less than linearly with the (inverse) transport rate. This is due to the fact that an increased fluid-to-wall mass transfer rate results in a larger steady state value of $C_{s}$, so that further fluid-to-wall mass transfer is counteracted, resulting in a less-than-linear increase of efficiency.

These data show that in order to attain a sizeable efficiency one needs to bring the wall temperature in the range $0.5 T_{a}<T_{s}<3 T_{a}$. Above this upper limit, the returns level off and below they are vanishingly small.

How long does it take to get into this range? To address this question a dynamic investigation is required, as we shall present in the next section.

\subsection{Dynamic Thermal Walls: $T_{s}$ free}

Next we simulate the situation in which the wall temperature changes in time based on the thermal transfer from the bulk flow. The conversion efficiency is controlled by the steady-state value of $T_{s} / T_{a}$, which in turn depends on the inlet fluid temperature $T_{i n}$. The results of a series of simulations with different values of $T_{a}$ are reported in figure 3 . 


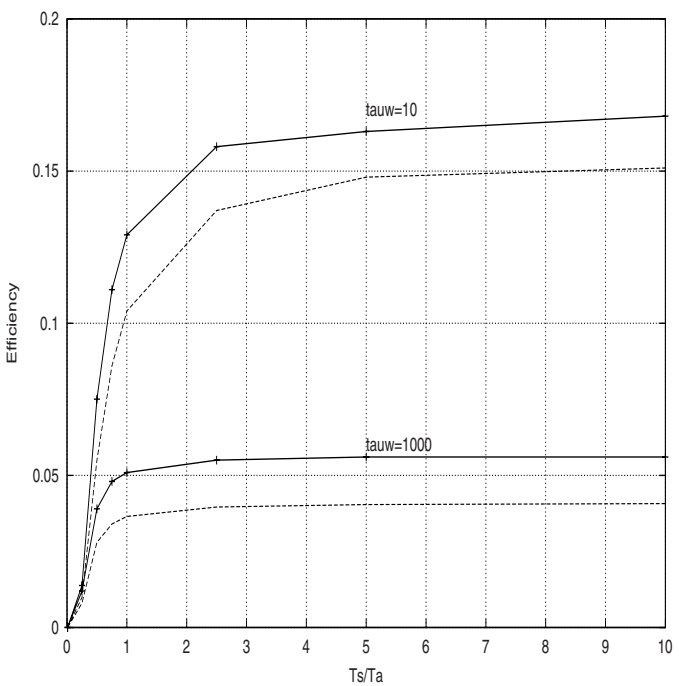

Fig. 2. Conversion efficiency as a function of the solid temperature $T_{s}$ (in units of activation temperature $T_{a}$ ) for two different absorption timescales $\tau_{w}=10,1000$. The chemical timescale is $\tau_{c}=100$. The thin lines indicate the values obtained by the analytical expression 13 .

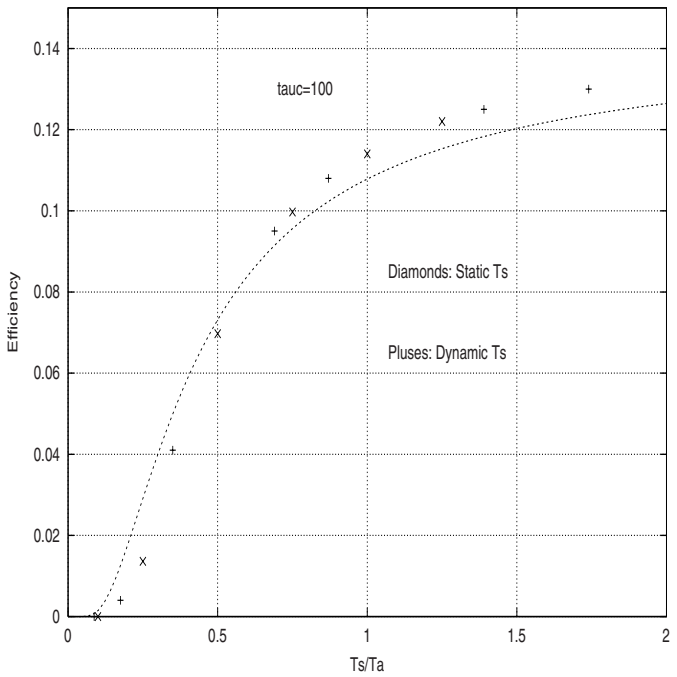

Fig. 3. Conversion efficiency as a function of the solid temperature $T_{s}$ (in units of $T_{a}$ ) for isothermal and dynamic solid temperature scenarios. Here $\tau_{w}=\tau_{c}=100$. The solid line corresponds to the analytical interpolation $1-e^{-0.22 / \sqrt{1+e^{1 / x}}}$, as suggested by the analytical expression (13). 
From this figure we see that indeed, the dynamic simulations fall within the same curve obtained in the static scenario. This is because the conversion efficiency only depends on the asymptotic value of $T_{s} / T_{a}$ regardless of the trajectory in time along which this steady state is attained. Note in fact that for the first-order chemical kinetics considered here the steady state is unique and independent of the initial conditions. An example of such thermal trajectories is shown in figure 4 , which reports the temperature history of two contiguous probe locations at $x=L / 2, y=L / 2, z=0$ (solid) and $x=L / 2, y=L / 2, z=1$ (fluid), for two distinct initial conditions $T_{s}(0)=T_{f}(0)=0.125$ and $T_{s}(0)=T_{f}(0)=1$ respectively.

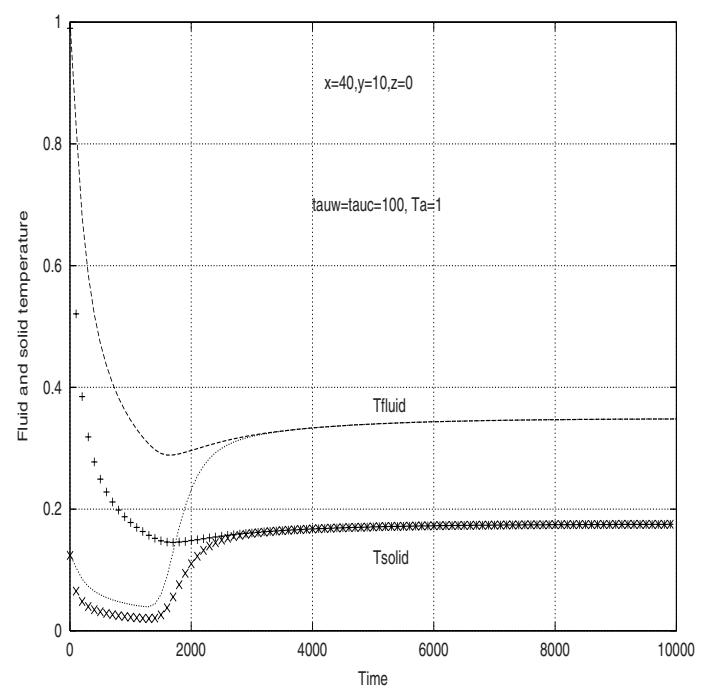

Fig. 4. Time evolution of the wall temperature at the location $x=40, y=10, z=0$ and the corresponding fluid temperature at $z=1$ (bottom wall) for a 'hot' start at $T=1$ and a 'cold' start at $T=0.125$. The main parameters are $\tau_{c}=\tau_{w}=100$.

This figure clearly shows that the time asymptotic states forget the initial conditions on a time scale of the order of a few thousands time steps, corresponding to a few advection times $t_{A}$. In physical units, this is about 2 milliseconds.

It is also interesting to observe the effect of raising the speed of chemical reactions by lowering the activation temperature threshold $T_{a}$. In Figure 5 we report the time evolution of the fluid and wall concentrations for $T_{a}=1$ (upper) and $T_{a}=0.1$ (lower).

Since the asymptotic ratio is given by $C_{f}^{\infty} / C_{s}^{\infty}=1+\tau_{w} / \tau_{c}$, it is clear that at lower activation temperatures the chemical timescale $\tau_{c}$ decreases, so that a gap opens up between the fluid and wall concentrations. The effect on the overall efficiency is dramatic, from $\eta=0.004$ to $\eta=0.13$, reflecting the width of the gap in concentrations. 


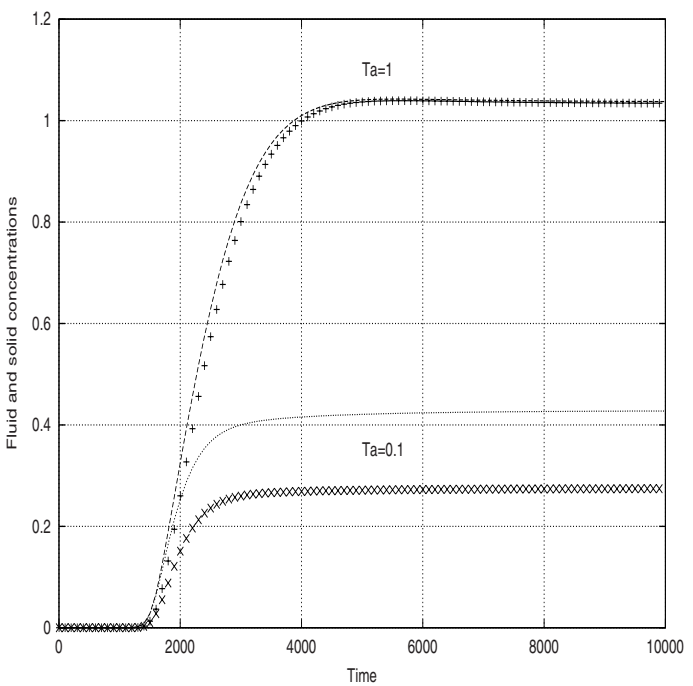

Fig. 5. Time evolution of the pollutant concentration at the bottom wall $(x=40, y=$ $10, z=0)$ and corresponding fluid locations $(z=1)$ for two distinct activation energies $T_{a}=0.1$ and $T_{a}=1$. Solid line: fluid. Plusses and crosses: solid. The main parameters are $\tau_{c}=\tau_{w}=100$.

\section{Summary and Future Developments}

Since it ticks at very small timesteps, the present approach is well suited to compute transient effects, and particularly, the time needed to attain significant chemical activity (light-off time). A number of future developments can be envisaged. First, one could use the present tool for geometrical design purposes, in order to investigate the effects of microcorrugations on the overall efficiency [4] with temperature dynamics taken into account. Another research direction is the study of the effects of more complex chemical kinetics, as well as effects of thermal coupling between the solid cells.

\section{References}

1. Whitesides, G., Stroock, A.D.: Flexible methods for microfluidics, Phys. Today,

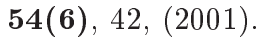

2. Oran E., Boris, J.: Numerical simulation of reactive flows, Elsevier Science, New York, (1987).

3. Benzi, R., Succi, S., Vergassola M.: The lattice Boltzmann equation: Theory and Applications, Phys. Rep. 222, (1992), 145-197, Wolfe-Gladrow, D.A.: Lattice Gas Cellular Automata and Lattice Boltzmann models, Springer Verlag, (2000), Succi, S., The Lattice Boltzmann equation, Oxford University Press, Oxford, (2001).

4. Succi, S., Smith, G., Gabrielli, A., Kaxiras, E.: Chemical efficiency of reactive microflows with heterogeneus catalysis: a lattice Boltzmann study, Europ. Phys. J.: Appl. Phys. 16(1), (2001), 71-84. 
5. Succi, S., Filippova, O., Smith, G., Kaxiras, E.: Applying the Lattice Boltzmann equation to multiscale fluid problems, Comp. in Science and Eng., 3(6), (2001), 2637.

6. Gabrielli, A., Succi, S., Kaxiras, E.: A lattice Boltzmann study of reactive microflows, Comp. Phys. Comm., 147, (2002), 516-521.

7. Succi, S., Chen, H., Teixeira, C., De Maio, A., Molvig, K., Bella, G.: An integer realization of the Lax scheme for the transport of multiple components, J. Comp. Phys., 152 , (1999), 493-516.

8. Qian, Y., d'Humières, D., Lallemand, P.: Lattice BGK models for the Navier-Stokes equation, Europhys. Lett. 17 , (1992), 479-484.

9. Lallemand, P., Luo, L.S.: Hybrid Finite-Difference thermal Lattice Boltzmann equation, Proceedings of the 11th Conference on Discrete Simulation of Fluid Dynamics and Soft-Condensed Matter, Shanghai, August 2002, to appear in Int. J. Mod. Phys. B.

10. Succi, S., Adamo, M., Bella, G., Bernaschi, M.: Multi-representation techniques for multiscale problems: Reactive microflows in a catalytic converter, Molecular Simulation, 25, 1-2, (2000), 13-26. 